\title{
Correlation and current anomalies in helical quantum dots
}

\author{
C. De Beule, ${ }^{1,{ }^{*}}$ N. Traverso Ziani, ${ }^{2,}{ }^{\dagger}$ M. Zarenia, ${ }^{1}$ B. Partoens, ${ }^{1}$ and B. Trauzettel ${ }^{2,3}$ \\ ${ }^{1}$ Department of Physics, University of Antwerp, 2020 Antwerp, Belgium \\ ${ }^{2}$ Institute of Theoretical Physics and Astrophysics, University of Würzburg, 97074 Würzburg, Germany \\ ${ }^{3}$ Department of Physics, University of California, Berkeley, California 94720, USA \\ (Received 7 January 2016; revised manuscript received 7 July 2016; published 7 October 2016)
}

\begin{abstract}
We theoretically investigate the ground-state properties of a quantum dot defined on the surface of a strong three-dimensional time-reversal invariant topological insulator. Confinement is realized by ferromagnetic barriers and Coulomb interaction is treated numerically for up to seven electrons in the dot. Experimentally relevant intermediate interaction strengths are considered. The topological origin of the dot has several consequences: (i) spin polarization increases and the ground state exhibits quantum phase transitions at specific angular momenta as a function of interaction strength, (ii) the onset of Wigner correlations takes place mainly in one spin channel, and (iii) the ground state is characterized by a robust persistent current that changes sign as a function of the distance from the center of the dot.
\end{abstract}

DOI: 10.1103/PhysRevB.94.155111

\section{INTRODUCTION}

Topological insulators (TIs) [1-7] are peculiar insulators hosting metallic states at boundaries with other insulators that are distinguished by band topology. The metallic interface states are, for a certain class of TIs, protected from timereversal invariant perturbations and characterized by spinmomentum locking [8,9]. Due to their special properties, the surface states are potentially interesting for applications in spintronics [10-12] and quantum computation [13,14]. A promising step toward device applications is represented by the progress made in topological insulator nanostructures. On the one hand, nanostructures can overcome some intrinsic limitations of current TIs, e.g., bulk transport [15-18]. On the other hand, the ability to produce topological nanostructures may lead to new physics, related, for instance, to Majorana fermions [19-21], parafermions [22-24], qubits [25], topological Josephson junctions [26-28], and anomalous superconducting pairing [29].

The main challenge in the theoretical description of nanostructured TIs can be identified with the need to properly take into account electron-electron interactions. Interaction effects in two-dimensional quantum spin Hall systems are striking: In the weakly interacting regime, the low-energy properties of the edge states are well described by the helical Luttinger liquid [30,31]; in the case of strong interactions, the physics is even richer, in terms of both exotic edge [22,23,32] and bulk [33] properties. For three-dimensional time-reversal invariant TIs, the Landau theory of the two-dimensional helical surface states has been developed to deal with the weak to intermediate interaction regime [34], while the strongly interacting regime is dominated by fractionalization [33].

Moreover, the study of interacting massless Dirac fermions, relevant for the surface of strong TIs, can greatly benefit from the corresponding research on the electronic properties of graphene [35]. For example, bulk graphene is characterized by the absence of Wigner crystallization [36]: The kinetic energy

\footnotetext{
*christophe.debeule@uantwerpen.be

†niccolo.traverso@physik.uni-wuerzburg.de
}

has the same density dependence as the Coulomb energy due to the linear spectrum, so there is no competition between these energy scales. In finite structures, such as quantum dots, this simple picture fails, and Wigner molecules, or their onset, may emerge for strong but reasonable interaction strengths [37,38]. A similar increase of the effects of electron-electron interactions in finite structures has been reported [39] for quantum dots in a two-dimensional electron gas (2DEG) [40-43]. In general, the same behavior also characterizes the surface states of strong three-dimensional TIs. However, as we show below, the presence of a single Dirac cone has remarkable consequences on the ground state of helical quantum dots.

In this paper, we investigate the ground-state properties of a circular quantum dot hosted on the surface of a threedimensional strong topological insulator, confined by hard wall (i.e., infinite mass) boundaries. The inspection is carried out numerically with exact diagonalization for up to seven electrons in the dot. We consider an intermediate interaction regime such that electrons are strongly correlated but the Wigner molecule is not fully developed, meaning that the electrons are not fully localized.

The paper is further organized as follows: In Sec. II, we introduce the model for the quantum dot. We discuss our results for the anomalous spin-resolved behavior of the ground state in Sec. III. In Sec. III A, we show that the quantum dot is characterized by an induced spin polarization that increases with interaction strength, similar to quantum dots in a 2DEG with strong Rashba coupling [44]. We draw an analogy, in terms of quantum phase transitions, between interactions in our system and external magnetic fields in 2DEG quantum dots. Moreover, the spatial distribution of the spin-resolved density shows a partial segregation between spins. While the density of the majority spin (spin up in our case) is localized mostly near the boundary of the dot, that of the minority spin (spin down in our case) is localized more near the center. Intuitively, this suggests that spin-down particles are effectively stiffer toward electron-electron interactions and, hence, energetically unfavored. This picture is supported by the spin-resolved density-density correlation functions, shown in Sec. IIIB. The majority spin component shows strong correlations, while the minority spin density remains more 
liquid-like. Finally, in Sec. III C, we find that the many-body ground state is characterized by a robust persistent current that changes sign as a function of the radius of the dot in all cases under consideration. In conclusion, we give a summary of our results and possible experimental realizations in Sec. IV.

\section{MODEL}

The Hamiltonian $H$ we consider is

$$
H=\sum_{i} H_{0}^{(i)}+V_{C}, \quad H_{0}^{(i)}=T^{(i)}+U^{(i)} .
$$

Here, $H_{0}$ is the one-particle Hamiltonian and $V_{C}$ the Coulomb interaction. For the one-particle wave functions, we adopt the spinor basis $\psi\left(\boldsymbol{r}^{(i)}\right)=\left(\psi_{+}\left(\boldsymbol{r}^{(i)}\right),-i \psi_{-}\left(\boldsymbol{r}^{(i)}\right)\right)^{T}$, where the coordinate $\boldsymbol{r}^{(i)}=\left(x^{(i)}, y^{(i)}\right)$ represents the position of the $i$ th electron on the TI surface. In this basis, the kinetic term $T^{(i)}$ reads $T^{(i)}=v_{F} \boldsymbol{\sigma}^{(i)} \cdot \boldsymbol{p}^{(i)}$, where $v_{F}$ is the Fermi velocity, $\boldsymbol{\sigma}^{(i)}=\left(\sigma_{x}^{(i)}, \sigma_{y}^{(i)}\right)$ are the Pauli matrices acting on the spin of the $i$ th particle, and $\boldsymbol{p}^{(i)}=-i \hbar \nabla_{\boldsymbol{r}^{(i)}}$. The confining potential $U^{(i)}$ we consider is $U^{(i)}=M \Theta\left(r^{(i)}-R\right) \sigma_{z}^{(i)}$, where $r^{(i)}=\left|\boldsymbol{r}^{(i)}\right|$, $\Theta(x)$ is the Heaviside step function, $R$ is the radius of the dot, and the limit $M \rightarrow \infty$ is taken for simplicity [45]. Note that this confinement necessarily breaks time-reversal symmetry. Experimentally, this could be realized by depositing a structured ferromagnetic layer on the TI surface. The (spinor) eigenvectors $\psi_{n, m}\left(\boldsymbol{r}^{(i)}\right)$ of $H_{0}^{(i)}$ are

$$
\psi_{n, m}(\boldsymbol{r})=A_{n, m} e^{i m \theta}\left(\begin{array}{c}
J_{m}\left(k_{n, m} r\right) \\
i e^{i \theta} J_{m+1}\left(k_{n, m} r\right)
\end{array}\right) .
$$

Here, $m$ is an integer, $\theta=\arctan (y / x), J_{m}(x)$ are Bessel functions, and $k_{n, m}$ are the solutions for $k$ with $n$ radial nodes in $\psi_{+}$, of the hard wall boundary condition

$$
J_{m}(k R)=J_{m+1}(k R),
$$

The spinor normalization then reads $A_{n, m}^{-2}=2 \pi R^{2} J_{m}$ $\left(k_{n, m} R\right)^{2}\left[2-(2 m+1) /\left(k_{n, m} R\right)\right]$ and the corresponding eigenvalues are $E_{n, m}=\hbar v_{F} k_{n, m}$. The spectrum is symmetric with respect to zero energy and a gap $\Delta \approx 2.87 \hbar v_{F} / R$ is present. Throughout this work, similar to Refs. [37,46-48], we assume all negative energy states to be filled and inert. For $(n, m)$ corresponding to positive energy, we define the fermionic annihilation (creation) operators $c_{n, m}\left(c_{n, m}^{\dagger}\right)$. The corresponding Fermi spinor $\Psi(\boldsymbol{r})$ is given by $\Psi(\boldsymbol{r})=\left(\left(\Psi_{+}(\boldsymbol{r}), \Psi_{-}(\boldsymbol{r})\right)^{T}=\right.$ $\sum_{n, m} \psi_{n, m}(\boldsymbol{r}) c_{n, m}$. Once the negative energy states are removed from the Hilbert space, the Hamiltonian becomes (repeated indexes are summed)

$$
\begin{aligned}
& H^{\prime}=H_{0}^{\prime}+V_{C}^{\prime}, \\
& H_{0}^{\prime}=E_{n, m} c_{n, m}^{\dagger} c_{n, m}, \\
& V_{C}^{\prime}=V_{n_{1}, m_{1}, n_{2}, m_{2}}^{n_{3}, m_{3}, n_{4}, m_{4}} c_{n_{1}, m_{1}}^{\dagger} c_{n_{2}, m_{2}}^{\dagger} c_{n_{3}, m_{3}} c_{n_{4}, m_{4}},
\end{aligned}
$$

where $V_{n_{1}, m_{1} ; n_{2}, m_{2}}^{n_{3}, m_{3} ; n_{4}, m_{4}}$ are the Coulomb matrix elements with respect to the spinor wave functions $\psi_{n_{i}, m_{i}}\left(\boldsymbol{r}^{(i)}\right)$. Since the total angular momentum $J_{z}=\sum_{i}\left(m_{i}+1 / 2\right)$ is conserved in our model, the Hamiltonian can be written in block diagonal form. The effective fine-structure constant $\alpha$ that controls the relative weight of kinetic and Coulomb energy is given by $\alpha=e^{2} /\left(4 \pi \epsilon_{0} \epsilon \hbar v_{F}\right)$, where $\epsilon$ is the relative permittivity of the TI surface. As expected, $\alpha$ is independent of the density, and depends only on $v_{F}$ and $\epsilon$. For $\mathrm{Bi}_{2} \mathrm{Te}_{3}$ thin films [49] on $\mathrm{SiO}_{2}$ [50], we estimate $\alpha \approx 1.8$, which could be increased further by strain or substrate engineering [51] to reduce $v_{F}$ or $\epsilon$, respectively. Moreover, strongly interacting electrons are to be expected on the surfaces of TIs with a strongly interacting bulk such as Heusler compounds, whose bulk electrons show heavy fermion behavior [52].

\section{RESULTS}

Our aim is to characterize the ground-state properties of a dot containing $N$ electrons, described by the Hamiltonian in Eq. (4), in the intermediate interaction regime $0<\alpha<2$. To do so, the ground-state averages $\langle O\rangle$ of operators $O$ are evaluated numerically by exact diagonalization.

\section{A. Spin polarization}

The first quantity we address is the spin polarization $\left\langle S_{z}\right\rangle=$ $\frac{\hbar}{2} \int d^{2} \boldsymbol{r}\left\langle\Psi^{\dagger}(\boldsymbol{r}) \sigma_{z} \Psi(\boldsymbol{r})\right\rangle$. A finite spin polarization $\left\langle S_{z}\right\rangle$, in the noninteracting case, is due to the presence of the boundary that breaks time-reversal symmetry. Turning on electron-electron interactions increases the spin polarization. Two different cases are shown in Fig. 1(a). For $N=4$, the increase is smooth, while for $N=7$, the increase is larger and shows a jump. As shown in Fig. 1(b), the difference is due to the nature of the ground state as a function of $\alpha$ : While for $N=4$, the total angular momentum is $J_{z}=4$ for $0<\alpha<2$, the jump in $\left\langle S_{z}\right\rangle$ for $N=7$ corresponds to a level crossing where $J_{z}$ changes from $J_{z}=6.5$ to $J_{z}=10.5$ at $\alpha \approx 0.7$. The physical significance of the spin polarization is the following: With increasing interaction strength, the system evolves toward a classical state: the Wigner molecule. In nontopological systems, the classical nature, and the consequent loss of the spin degree of freedom, is realized by the fact that many states tend to become degenerate in the limit of infinite interaction strength. The presence of a single Dirac cone with spin-momentum locking, however, prevents our system from developing this degeneracy. Increasing the spin polarization is therefore a natural way to approach the classical limit of a collective spinless (or spin-polarized) system in the helical quantum dot. The quantum phase diagram shown in Fig. 1(b) indicates that the effect is generic for $2<N<7$ and $0<\alpha<2$. Moreover,
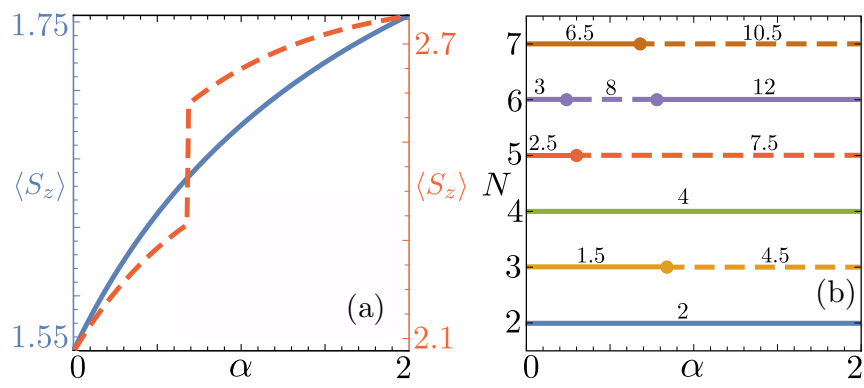

FIG. 1. (a) Spin polarization $\left\langle S_{z}\right\rangle$, in units $\frac{\hbar}{2}$, as a function of $\alpha$ for $N=4$ (blue, solid, left $y$ axis), and $N=7$ (red, dashed, right $y$ axis). (b) Quantum phase diagram as a function of $N$ and $\alpha$. Dots indicate phase transitions and the numbers are the angular momenta $J_{z}$ of the ground state. 

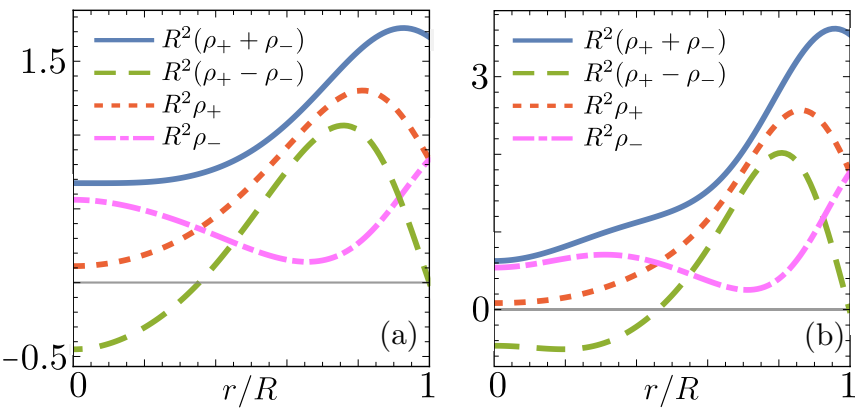

FIG. 2. Total electron density $\rho_{+}(r)+\rho_{-}(r)$ (blue, solid), spin imbalance $\rho_{+}(r)-\rho_{-}(r)$ (green, dashed), $\rho_{+}(r)$ (red, dotted), and $\rho_{-}(r)$ (purple, dash-dotted) as a function of the radius $r$ for $\alpha=2$ and (a) $N=4$, (b) $N=7$.

it contains further peculiarities: In ordinary 2DEG quantum dots, $\left\langle S_{z}\right\rangle$ does not change with interaction strength. In this case, the total spin is fixed by an applied magnetic field which itself can induce quantum phase transitions toward states with a higher spin imbalance $[39,40]$. The magic numbers, i.e., the orbital angular momenta $M$ of the ground state, can then be determined from the symmetry properties of the wave function (e.g., $M=3,6, \ldots$ for $N=3$ and $M=$ $6,10, \ldots$ for $N=4$ ) [53]. The interplay of spin-momentum locking, Coulomb interaction, and the boundary, which acts as a spin-selection mechanism, yields similar (but different) quantum phase transitions in our system at zero magnetic field as $\alpha$ is increased. Most strikingly, the analogy is profound: The observed $J_{z}$ ground-state values can be obtained from assuming a maximum value for $\left\langle S_{z}\right\rangle$, and the same magic number rule for the angular momentum expectation value.

More can be learned about the spin structure by inspecting the radial distribution of the spin-up and spin-down densities $\rho_{ \pm}(r)=\left\langle\Psi^{\dagger}(\boldsymbol{r})\left[\left(1 \pm \sigma_{z}\right) / 2\right] \Psi(\boldsymbol{r})\right\rangle$. For $N=4,5,6,7$ (the cases $N=4$ and $N=7$ are shown in Fig. 2 for $\alpha=2$ ) the spin-down component is dominant close to the center, while the spin-up component is dominant closer to the edge. Directly at the edge, however, the spin-resolved densities are forced to be equal by the boundary conditions. Qualitatively, this means that the spin-down density is stiffer toward interactions because it is concentrated closer to the center than the spin-up density. This behavior is consistent with the increase of the spin polarization $\left\langle S_{z}\right\rangle$ when the interaction strength is increased: Spin-up electrons respond faster to electronelectron interactions, and therefore it is energetically favorable to polarize the system.

\section{B. Spin-resolved correlations}

To directly access the spin selection, we address the spinresolved density-density correlation functions $d_{s, s^{\prime}}\left(\boldsymbol{r}, \boldsymbol{r}^{\prime}\right)$, with $s, s^{\prime}= \pm$ labeling the spin projection. Explicitly, we define

$$
d_{s, s^{\prime}}\left(\boldsymbol{r}, \boldsymbol{r}^{\prime}\right)=\frac{\left\langle\Psi_{s}^{\dagger}(\boldsymbol{r}) \Psi_{s^{\prime}}^{\dagger}\left(\boldsymbol{r}^{\prime}\right) \Psi_{s^{\prime}}\left(\boldsymbol{r}^{\prime}\right) \Psi_{s}(\boldsymbol{r})\right\rangle}{N\left(N-\delta_{s, s^{\prime}}\right)},
$$

so that the interpretation of $d_{s, s^{\prime}}\left(\boldsymbol{r}, \boldsymbol{r}^{\prime}\right)$ is the probability of finding a particle with spin projection $s$ at position $r$ if an electron with spin projection $s^{\prime}$ is fixed at position $\boldsymbol{r}^{\prime}$. The results for $N=4$ and $N=7$ (which well represent the physics of up to
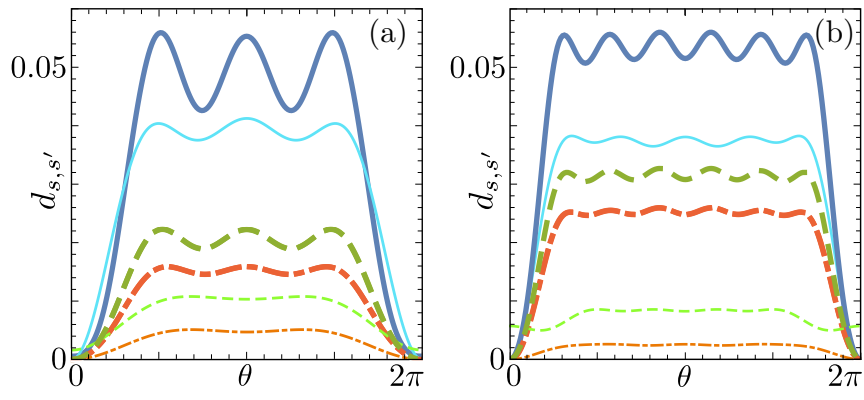

FIG. 3. $d_{+,+}\left(\boldsymbol{r}, \boldsymbol{r}^{\prime}\right)$ (blue, solid), $d_{+,-}\left(\boldsymbol{r}, \boldsymbol{r}^{\prime}\right)$ (green, dashed), and $d_{-,-}\left(\boldsymbol{r}, \boldsymbol{r}^{\prime}\right)$ (red, dash-dotted) as functions of $\theta$; light thin lines correspond to $\alpha=0$ and dark thick lines to $\alpha=2$. (a) $N=4, \theta^{\prime}=0$, and $r=r^{\prime}=0.8 R$ for $\alpha=0$, while $r=r^{\prime}=0.93 R$ for $\alpha=2$. (b) $N=7, \theta^{\prime}=0$, and $r=r^{\prime}=0.76 R$ for $\alpha=0$, while $r=r^{\prime}=0.96 R$ for $\alpha=2$.

seven particles) are shown in Fig. 3 in the noninteracting case $\alpha=0$ and for $\alpha=2$. In the figure, $r$ and $r^{\prime}$ are fixed at the maximum of the total density (spin-up density for $N=7$ and $\alpha=0)$ and $d_{s, s^{\prime}}\left(\boldsymbol{r}, \boldsymbol{r}^{\prime}\right)$ is plotted as a function of the polar angle $\theta$ of $\boldsymbol{r}$ with $\theta^{\prime}=0$. In the noninteracting case, we observe that $d_{+,+}\left(\boldsymbol{r}, \boldsymbol{r}^{\prime}\right)$ dominates due to the spin polarization induced by the boundary. Moreover, it is characterized by three and six peaks for respectively $N=4$ and $N=7$, which means that, on average, the weight of the spin-up component of each electron is dominant. Consistent with the Pauli principle, $d_{+,+}\left(\boldsymbol{r}, \boldsymbol{r}^{\prime}\right)$ and $d_{-,-}\left(\boldsymbol{r}, \boldsymbol{r}^{\prime}\right)$ are zero for $\theta=0$, while $d_{+,-}\left(\boldsymbol{r}, \boldsymbol{r}^{\prime}\right)$ is not. When interactions are increased the peaks in $d_{+,+}\left(\boldsymbol{r}, \boldsymbol{r}^{\prime}\right)$ become more pronounced, indicating the onset of Wigner oscillations. The correlation function $d_{+,-}\left(\boldsymbol{r}, \boldsymbol{r}^{\prime}\right)$ exhibits respectively three and six (weaker) oscillations as well. Remarkably, no signature of antiferromagnetic correlations, characterizing conventional 2DEG dots [54], are present. This is a consequence of the topological origin of the system. We also find $d_{+,-}\left(\boldsymbol{r}, \boldsymbol{r}^{\prime}\right) \sim 0$ at $\theta=0$ for $\alpha=2$, indicating unambiguously that $\alpha=2$ corresponds to significant interactions in the dot. The spindown correlations $d_{-,-}\left(\boldsymbol{r}, \boldsymbol{r}^{\prime}\right)$, however, remain suppressed (liquid-like) even for $\alpha=2$. The system is hence characterized by a spin-selective Wigner oscillation. This is consistent with both the increase in spin polarization and the radial dependence of the spin-resolved density. Intuitively speaking, one of the effects of interactions in confined systems is to push particles closer to the boundaries so as to minimize the interaction energy. Particles that remain near the center when interactions are increased (the spin-down density in this case) do not minimize the interaction energy, and their spatial distribution and correlations are still mostly determined by kinetic energy and confinement. Therefore, a more strongly polarized state is favored with increasing interaction strength.

\section{Persistent current}

In the last part of this work, we inspect the local azimuthal current density

$$
J_{\theta}(r)=e v_{F} \hat{\theta} \cdot\left\langle\Psi^{\dagger}(\boldsymbol{r}) \boldsymbol{\sigma} \Psi(\boldsymbol{r})\right\rangle,
$$

where $\hat{\theta}=(-y / r, x / r)$. Notably, the current density can be identified with a magnetization current through the relation $\boldsymbol{J}=\boldsymbol{\nabla} \times \boldsymbol{M}$ via a suitably defined orbital magnetization $\boldsymbol{M}$. 

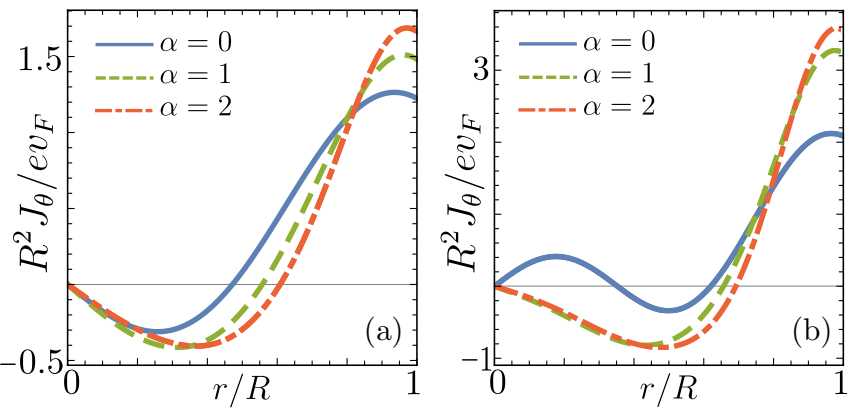

FIG. 4. Azimuthal current density $J_{\theta}(r)$ of the ground state for $\alpha=0, \alpha=1$, and $\alpha=2$ for (a) $N=4$ and (b) $N=7$.

Furthermore, it is also directly proportional to the spin density which is peculiar to Dirac surface states. The contribution of the spin magnetization to the current can be neglected for a dot on the surface of $\mathrm{Bi}_{2} \mathrm{Se}_{3}$, whose topological surface state has a $g$-factor of 18 [55], with radius $R \gtrsim 100 \mathrm{~nm}$ since it scales as $R^{-3}$ while $J_{\theta}(r)$ goes as $R^{-2}$. Note that $J_{\theta}(r)$ is not a spin-resolved observable but depends crucially on the distribution of orbital angular momenta which are related to the spin degree of freedom by spin-momentum locking. A peculiar behavior of $J_{\theta}(r)$ can thus be expected. In all cases, the total angular momentum of the ground state is nonzero such that the ground state is a rotating Wigner oscillation. However, due to spin-momentum locking, the orbital angular momentum is not a good quantum number and the rotation pattern is nontrivial. As shown in Fig. 4 for the cases (a) $N=4$ and (b) $N=7$, $J_{\theta}(r)$ changes sign as a function of $r$ with positive currents close to the edge and negative currents closer to the center. This behavior is due to a change of the contributing angular momenta to the ground state as a function of $r$, and goes beyond the intuitive picture of a coherently rotating Wigner molecule. Moreover, the boundary between regions of opposite current becomes sharper with increasing interaction strength.

In graphene [56] and other systems [57], such currents can also appear if time-reversal symmetry is broken; however, they are not robust against disorder. For instance, in graphene, states with a different valley degree of freedom can easily be coupled by rough boundaries, which strongly influences the persistent current.

\section{SUMMARY AND CONCLUSIONS}

We have analyzed the ground-state properties of a quantum dot defined on the surface of a three-dimensional strong topological insulator. We considered an intermediate interaction regime characterized by the onset of Wigner oscillations in the density-density correlation functions. The onset of Wigner oscillations is special in our system: It is concomitant with an increase of the spin polarization of the dot, so that electron-electron interactions in the helical dot play a similar role as an external magnetic field for 2DEG quantum dots, and they affect mainly one spin component. Finally, we discussed the behavior of the azimuthal particle current which changes sign as a function of the distance from the center of the dot, revealing a fingerprint of spin-momentum locking and hence of the topological origin of the system.

As far as the experimental observations of our predictions are concerned, different issues might arise. First, the realization of quantum dots on the surface of topological insulators remains a challenge but it should, for instance, be possible by magnetic confinement. Second, the spin polarization that we predict can, in principle, be accessed by coupling the dot to spin-polarized local probes $[58,59]$. The texture characterizing $J_{\theta}(r)$ can be detected with nitrogen-vacancy (NV) centers in diamonds or nano-size superconducting quantum interference devices (nanoSQUIDs) [60-62]. In fact, for a dot with radius $R=500 \mathrm{~nm}$, a classical finite-element calculation shows that the typical variations of the resulting magnetic field are of the order of $0.2 \mu T$, which is measurable by both techniques. In smaller dots, the signal, which scales as $1 / R^{2}$, is larger, but in that case the required spatial resolution favors the use of $\mathrm{NV}$ centers.

\section{ACKNOWLEDGMENTS}

We thank F. Cavaliere, F. Crépin, C. Felser, and B. Yan for interesting discussions, and $\mathrm{S}$. Curreli for performing the finite-element calculation of the magnetic field in COMSOL. C.D.B. and M.Z. are supported by the Flemish Research Foundation (FWO). N.T.Z. and B.T. acknowledge financial support by the DFG (SPP1666 and SFB1170 "ToCoTronics"), the Helmholtz Foundation (VITI), and the ENB Graduate School on "Topological Insulators."
[1] C. L. Kane and E. J. Mele, Phys. Rev. Lett. 95, 226801 (2005).

[2] B. A. Bernevig, T. L. Hughes, and S.-C. Zhang, Science 314, 1757 (2006).

[3] M. König, S. Weidmann, C. Brüne, A. Roth, H. Buhmann, L. W. Molenkampf, X.-L. Qi, and S.-C. Zhang, Science 318, 766 (2007).

[4] I. Knez, R.-R. Du, and G. Sullivan, Phys. Rev. Lett. 107, 136603 (2011).

[5] L. Fu, C. L. Kane, and E. J. Mele, Phys. Rev. Lett. 98, 106803 (2007).

[6] L. Fu and C. L. Kane, Phys. Rev. B 76, 045302 (2007).

[7] D. Hsieh, Y. Xia, D. Qian, L. Wray, J. H. Dil, F. Meier, J. Osterwalder, L. Patthey, J. G. Checkelsky, N. P. Ong, A. V.
Fedorov, H. Lin, A. Bansi, D. Grauer, Y. S. Hor, R. J. Cava, and M. Z. Hasan, Nature 452, 970 (2008).

[8] M. Z. Hasan and C. L. Kane, Rev. Mod. Phys. 82, 3045 (2010).

[9] X.-L. Qi and S.-C. Zhang, Rev. Mod. Phys. 83, 1057 (2011).

[10] D. Pesin and A. H. Macdonald, Nat. Mater. 11, 409 (2012).

[11] A. Roth, C. Brüne, H. Buhmann, L. W. Molenkamp, J. Maciejko, X.-L. Qi, and S.-C. Zhang, Science 325, 294 (2009).

[12] P. Michetti and B. Trauzettel, Appl. Phys. Lett. 102, 063503 (2013).

[13] S. Das Sarma, M. Freedman, and C. Nayak, Phys. Today 59, 32 (2006).

[14] C. Nayak, S. H. Simon, A. Stern, M. Freedman, and S. Das Sarma, Rev. Mod. Phys. 80, 1083 (2008). 
[15] M. König, M. Baenninger, A. G. F. Garcia, N. Harjee, B. L. Pruitt, C. Ames, P. Leubner, C. Brüne, H. Buhmann, L. W. Molenkamp, and D. Goldhaber-Gordon, Phys. Rev. X 3, 021003 (2013).

[16] D. Kong, J. C. Randel, H. Peng, J. J. Cha, S. Meister, K. Lai, Y. Chen, Z.-X. Shen, H. C. Manoharan, and Y. Cui, Nano Lett. 10, 329 (2009).

[17] D. Kong and Y. Cui, Nat. Chem. 3, 845 (2011).

[18] S. Cho, D. Kim, P. Syers, N. P. Butch, J. Paglione, and M. S. Fuhrer, Nano Lett. 12, 469 (2012).

[19] L. Fu and C. L. Kane, Phys. Rev. Lett. 100, 096407 (2008).

[20] L. Fu and C. L. Kane, Phys. Rev. Lett. 102, 216403 (2009).

[21] J. D. Sau, S. Tewari, R. Lutchyn, T. Stanescu, and S. Das Sarma, Phys. Rev. B 82, 214509 (2010).

[22] F. Zhang and C. L. Kane, Phys. Rev. Lett. 113, 036401 (2014).

[23] C. P. Orth, R. P. Tiwari, T. Meng, and T. L. Schmidt, Phys. Rev. B 91, 081406(R) (2015).

[24] J. Klinovaja, A. Yacoby, and D. Loss, Phys. Rev. B 90, 155447 (2014).

[25] G. J. Ferreira and D. Loss, Phys. Rev. Lett. 111, 106802 (2013).

[26] L. Fu and C. L. Kane, Phys. Rev. B 79, 161408(R) (2009).

[27] G. Tkachov and E. M. Hankiewicz, Phys. Rev. B 88, 075401 (2013).

[28] J. Wiedenmann, E. Bocquillon, R. S. Deacon, S. Hartinger, O. Herrmann, T. M. Klapwijk, L. Maier, C. Ames, C. Brüne, C. Gould, A. Oiwa, K. Ishibashi, S. Tarucha, H. Buhmann, and L. W. Molenkamp, Nat. Commun. 7, 10303 (2016).

[29] F. Crépin, P. Burset, and B. Trauzettel, Phys. Rev. B 92, 100507(R) (2015).

[30] C. Wu, B. A. Bernevig, and S.-C. Zhang, Phys. Rev. Lett. 96, 106401 (2006).

[31] C. Xu and J. E. Moore, Phys. Rev. B 73, 045322 (2006).

[32] N. T. Ziani, F. Crépin, and B. Trauzettel, Phys. Rev. Lett. 115, 206402 (2015).

[33] J. Maciejko and G. Fiete, Nat. Phys. 11, 385 (2015).

[34] R. Lundgren and J. Maciejko, Phys. Rev. Lett. 115, 066401 (2015).

[35] M. I. Katsnelson, Graphene: Carbon in Two Dimensions (Cambridge University, Cambridge, England, 2012).

[36] H. P. Dahal, Y. N. Joglekar, K. S. Bedell, and A. V. Balatsky, Phys. Rev. B 74, 233405 (2006).

[37] T. Paananen, R. Egger, and H. Siedentop, Phys. Rev. B 83, 085409 (2011).

[38] K. A. Guerrero-Becerra and M. Rontani, Phys. Rev. B 90, 125446 (2014).

[39] R. Egger, W. Hausler, C. H. Mak, and H. Grabert, Phys. Rev. Lett. 82, 3320 (1999).

[40] S. M. Reimann and M. Manninen, Rev. Mod. Phys. 74, 1283 (2002).
[41] C. Yannouleas and U. Landman, Rep. Prog. Phys. 70, 2067 (2007).

[42] S. Kalliakos, M. Rontani, V. Pellegrini, C. P. Garcia, A. Pinczuk, G. Goldoni, M. Molinari, L. N. Pfeiffer, and K. W. West, Nat. Phys. 4, 467 (2008).

[43] F. Cavaliere, U. De Giovannini, M. Sassetti, and B. Kramer, New J. Phys. 11, 123004 (2009).

[44] A. Naseri, A. Zazunov, and R. Egger, Phys. Rev. X 4, 031033 (2014).

[45] M. V. Berry and R. J. Mondragon, Proc. R. Soc. London A 412, 53 (1987).

[46] J. Sucher, Phys. Rev. 107, 1448 (1957); 109, 1010 (1958); Phys. Rev. A 22, 348 (1980); Int. J. Quantum Chem. 25, 3 (1984).

[47] W. Häusler and R. Egger, Phys. Rev. B 80, 161402(R) (2009).

[48] R. Egger, A. De Martino, H. Siedentop, and E. Stockmeyer, J. Phys. A: Math. Theor. 43, 215202 (2010).

[49] Y.-Y. Li, G. Wang, X.-G. Zhu, M.-H. Liu, C. Ye, X. Chen, Y.-Y. Wang, K. He, L.-L. Wang, X.-C. Ma, H.-J. Zhang, X. Dai, Z. Fang, X.-C. Xie, Y. Liu, X.-L. Qi, J.-F. Jia, S.-C. Zhang, and Q.-K. Xue, Adv. Mater. 22, 4002 (2010).

[50] S. K. Jerng, K. Joo, Y. Kim, S. M. Yoon, J. H. Lee, M. Kim, J. S. Kim, E. Yoon, S. H. Chun, and Y. S. Kim, Nanoscale 5, 10618 (2013).

[51] M. R. Baklanov and K. Maex, Philos. Trans. R. Soc. London A 364, 201 (2006).

[52] S. Chadov, X. Qi, J. Kübler, G. H. Fecher, C. Felser, and S. C. Zhang, Nat. Mater. 9, 541 (2010).

[53] W. Y. Ruan, Y. Y. Liu, C. G. Bao, and Z. Q. Zhang, Phys. Rev. B 51, 7942 (1995).

[54] S. M. Reimann, M. Koskinen, and M. Manninen, Phys. Rev. B 62, 8108 (2000).

[55] Y.-S. Fu, T. Hanaguri, L. Igarashi, M. Kawamura, M. S. Bahramy, and T. Sasagawa, Nat. Commun. 7, 10829 (2016).

[56] M. Grujić, M. Zarenia, A. Chaves, M. Tadić, G. A. Farias, and F. M. Peeters, Phys. Rev. B 84, 205441 (2011).

[57] J. Reijniers, F. M. Peeters, and A. Matulis, Phys. Rev. B 59, 2817 (1999).

[58] R. Wiesendanger, Rev. Mod. Phys. 81, 1495 (2009).

[59] M. Bode, Rep. Prog. Phys. 66, 523 (2003).

[60] P. Maletinsky, S. Hong, M. S. Grinolds, B. Hausmann, M. D. Lukin, R. L. Walsworth, M. Loncar, and A. Yacoby, Nat. Nanotech. 7, 320 (2012).

[61] M. S. Grinolds, S. Hong, P. Maletinsky, L. Luan, M. D. Lukin, R. L. Walsworth, and A. Yacoby, Nat. Phys. 9, 215 (2013).

[62] D. Vasyukov, Y. Anahory, L. Embon, D. Halbertal, J. Cuppens, L. Neeman, A. Finkler, Y. Segev, Y. Myasoedov, M. L. Rappaport, M. E. Huber, and E. Zeldov, Nat. Nanotech. 8, 639 (2013). 